\title{
Linking bottleneck clogging with flow kinematics in granular materials: The role of silo width
}

\author{
D. Gella, D. Maza, and I. Zuriguel \\ Departamento de Física y Matemática Aplicada, Facultad de Ciencias, Universidad de Navarra, \\ Pamplona, Spain
}

A. Ashour

Institute of Experimental Physics, Otto von Guericke University, 39106 Magdeburg, Germany and Faculty of Engineering and Technology, Future University, End of 90 Street, New Cairo, Egypt

R. Arévalo

Division of Physics and Applied Physics, School of Physical and Mathematical Sciences, Nanyang Technological University, 25 Nanyang Link, 637371, Singapore

R. Stannarius

Institute of Experimental Physics, Otto von Guericke University, 39106 Magdeburg, Germany (Received 20 February 2017; published 30 August 2017)

\begin{abstract}
We demonstrate experimentally that clogging in a silo correlates with some features of the particle velocities in the outlet proximities. This finding, that links the formation of clogs with a kinematic property of the system, is obtained by looking at the effect that the position of the lateral walls of the silo has on the flow and clogging behavior. Surprisingly, the avalanche size depends nonmonotonically on the distance of the outlet from the lateral walls. Apart from evidencing the relevance of a parameter that has been traditionally overlooked in bottleneck flow, this nonmonotonicity supposes a benchmark with which to explore the correlation of clogging probability with different variables within the system. Among these, we find that the velocity of the particles above the outlet and their fluctuations seem to be behind the nonmonotonicity in the avalanche size versus wall distance curve.
\end{abstract}

DOI: 10.1103/PhysRevFluids.2.084304

\section{INTRODUCTION}

When a many-body system passes through a bottleneck, the development of blockages may lead to flow intermittencies that eventually end up in a complete arrest of the flow. This clogging phenomenon appears in many different systems with typical sizes ranging over several orders of magnitude. At very small spatial scales, clogging leads to intermittent flow when a dense suspension of microbes [1] or microparticles [2-4] passes through a constriction in a microchannel. Clogging is also behind dramatic cases of deaths when crowds in panic are evacuated through small emergency exits [5]. On an intermediate scale, clogging of granular materials in narrow conduits and silos supposes an hindrance as its occurrence may completely arrest a production line.

Clogging in all these different systems shares strong similarities [6] and, in the past years, its noteworthy importance has lead to a significant advance in its understanding. This has been especially notable for the case of inert grains within a silo, a system which has been traditionally used as a reference to compare other, more complex, scenarios. Typically, clogging is characterized by avalanche size statistics, where avalanche sizes are defined as the number of beads that flow out

\footnotetext{
*iker@unav.es
} 


\section{GELLA et al.}

of the silo from the destruction of a clogging structure until the development of a new one. From the pioneering work of To in 2001 [7], we know that this magnitude grows abruptly with the outlet size [8-11]. It has also been proven that, for a given outlet size, the avalanche size distribution decays exponentially [12], indicating a constant probability of clogging over the whole avalanche duration [11-14]. Apart from these findings, the development of blockages was reported to be affected by the packing fraction above the orifice, which may determine the probability that a given number of particles meet above the outlet [15]. The placement of an obstacle above the orifice can prevent clogging if its position is appropriately selected [6]. Other variables that have been shown to affect clogging are orifice geometry [16,17], particle shape [18-20], particle polydispersity [21], and gravity [22-24]. Nevertheless, we are not aware of any work looking at the influence of the width of the silo, a variable that in most of the existing experiments and simulations remains uncontrolled.

In this work, we aim to explore the role of the lateral walls on silo clogging. To this end, we have performed experiments and simulations of a two-dimensional silo in which we set the outlet and particle size, and we vary the distance of the lateral walls to the orifice. Our findings reveal that this parameter affects the mean avalanche size in a complex manner, evidencing a correlation with some features of the flow of grains in the outlet proximities. This result suggests that the ambition of correlating clogging with a kinetic property of the flow [25] is achievable.

\section{EXPERIMENTAL SETUP}

The experimental apparatus consists of a two-dimensional rectangular silo similar to the one described in Ref. [10], as shown in Fig. 1. The silo is $800 \mathrm{~mm}$ high and is made of two glass sheets separated by two vertical stripes $1.0 \mathrm{~mm}$ thick that also act as lateral walls. The distance among these two stripes determines the width of the silo $W$. The bottom is flat, being formed by two facing metal pieces which are slightly separated, giving rise to the outlet. In this work, the outlet is always at the center of the silo $( \pm 0.2 \mathrm{~mm})$ and its size $(D=4.32 \pm 0.02 \mathrm{~mm})$ is fixed by gluing the metal pieces to the supporting bench. The granular sample consists on monodisperse stainless steel beads with mass $4.00 \pm 0.01 \mathrm{mg}$ and diameter of $d=1.00 \pm 0.01 \mathrm{~mm}$. These grains are poured into the silo along its whole width, through a hopper at the top, conforming a monolayer. After the silo filling, grains start to flow freely through the outlet until an arch is formed and stabilized. When this occurs, the avalanche size is measured, in number of beads, using a balance placed below the orifice. Then, the flow is restarted by blowing a jet of compressed air aimed at the orifice. The silo is refilled whenever the level of grains falls below $300 \mathrm{~mm}$. For the case of narrow silos, this $300-\mathrm{mm}$ level is kept constant by means of an intermediate hopper structure that allows a continuous refilling, which has been checked to have no effect on the development of clogging. In this way, around 2000 avalanches were registered for each of the 13 different values of $W$ investigated.

For several of these silo width configurations, 10 movies of the region above the outlet were recorded at 2000 frames per second during 4 s. Importantly, the videos were always recorded during the avalanche, avoiding flow interruptions. From these recordings we obtained the positions of the particles, which were thereafter used to calculate space-time averaged magnitudes $[26,27]$. Specifically, the two-dimensional solid fraction $\phi$ and velocity $v$ values were attained by implementing a coarse-graining technique [28,29]:

$$
\begin{gathered}
\phi(\vec{r}, t)=A_{P} \sum_{i=1}^{N} \Theta\left[\vec{r}-\vec{r}_{i}(t)\right], \\
\vec{v}(\vec{r}, t)=A_{P} \sum_{i=1}^{N} \vec{v}_{i} \Theta\left[\vec{r}-\vec{r}_{i}(t)\right] / \phi(\vec{r}, t),
\end{gathered}
$$

where $A_{P}$ is the two-dimensional (2D) projected area of the particles, $N$ is the number of them, and $\Theta\left[\vec{r}-\vec{r}_{i}(t)\right]=\left[1 / 2 \pi \omega^{2}\right] \exp \left(-\left|\vec{r}-\vec{r}_{i}(t)\right|^{2} / 2 \omega^{2}\right)$ is the coarse-graining function, a Gaussian whose $\mathrm{rms}, \omega$, was set to the particle radius in order to minimize the strong dependence of the averaging 

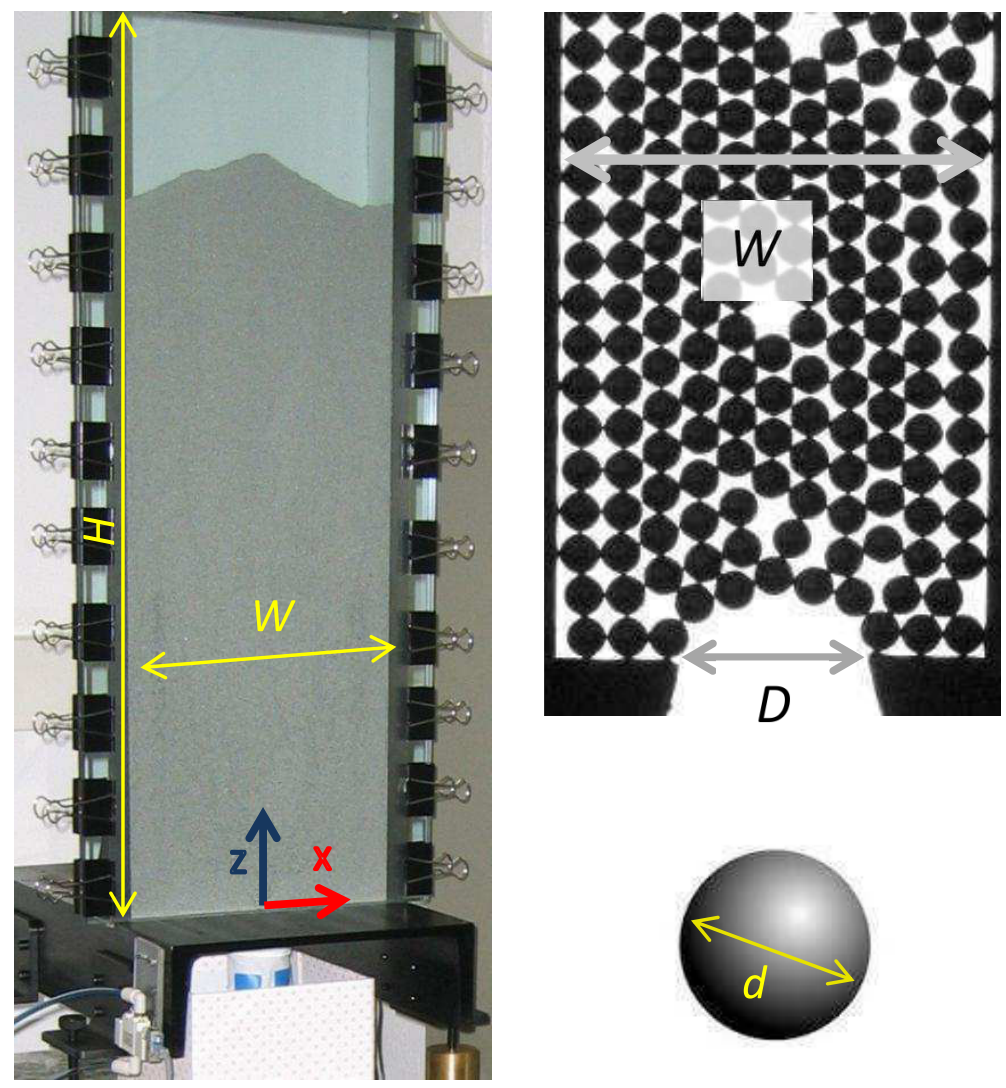

FIG. 1. Photographs of the two-dimensional silo. $W$ is the silo width; $D=4.32 \mathrm{~mm}$ is the outlet length; $d=1.00 \mathrm{~mm}$ is the particle's diameter; and $H=800 \mathrm{~mm}$ is the height of the silo. The origin of the Cartesian coordinate system has been set at the center of the exit orifice.

domain size observed for larger values [30]. Then, mean fields $\phi(\vec{r})$ and $\vec{V}(\vec{r})$ were calculated by time averaging throughout all the temporal sequences in each experimental condition. Analogously, a magnitude that accounts for the local velocity fluctuations, which is proportional to the trace of the recently introduced kinetic stress tensor [27], was computed in the two-dimensional projection as

$$
\sigma^{k}(\vec{r}, t)=A_{P} \sum_{i=1}^{N}\left(v_{i x}^{\prime 2}+v_{i z}^{\prime 2}\right) \Theta\left[\vec{r}-\vec{r}_{i}(t)\right],
$$

where $v_{i x}^{\prime}$ and $v_{i z}^{\prime}$ are the two components of the vector $\vec{v}^{\prime}\left[\vec{r}_{i}(t)\right]=\vec{v}\left[\vec{r}_{i}(t)\right]-\vec{V}\left[\vec{r}_{i}(t)\right]$. Finally, the temporal average of $\sigma^{k}(\vec{r}, t)$ is calculated, which will be named $\sigma^{k}(\vec{r})$.

In addition to the two-dimensional experiments, some tests have been implemented using threedimensional (3D) silos. The setup is the one already used in Refs. [8,12]. The silos were made with stainless steel cylinders $500 \mathrm{~mm}$ high and with different diameters $W_{3 D}$ (see Table I). The bottom of the silo is a flat disk with a circular hole in the center which, in this work, has a fixed diameter of $D_{3 D}=26.35 \pm 0.05 \mathrm{~mm}$. The particles were delrin spheres with mass $0.360 \pm 0.001 \mathrm{~g}$ and $d_{3 D}=8.00 \pm 0.01 \mathrm{~mm}$ diameter. Therefore, the rescaled outlet size is $D_{3 D} / d_{3 D}=3.29$. The experimental procedure is the same than for the two-dimensional case; i.e., a balance at the bottom detects the avalanche weight and a blower destroys the clogging arches to trigger a new avalanche. The silo is also refilled whenever the level of the grains falls below $300 \mathrm{~mm}$. 
TABLE I. Mean avalanche size (in number of particles) and 95\% confidence interval obtained in four threedimensional silos with different diameters $W_{3 D}$ and the same outlet size (diameter $D_{3 D}=26.35 \pm 0.05 \mathrm{~mm}$ ). The particle diameter is $d_{3 D}=8 \mathrm{~mm}$ so the rescaled outlet size is $D_{3 D} / d_{3 D}=3.29$.

\begin{tabular}{lcccc}
\hline \hline$W_{3 D}$ & $36 \mathrm{~mm}$ & $46 \mathrm{~mm}$ & $80 \mathrm{~mm}$ & $150 \mathrm{~mm}$ \\
\hline$W_{3 D} / d_{3 D}$ & 4.5 & 5.75 & 10 & 18.75 \\
$\langle s\rangle$ & $>10^{4}$ & 72.5 & 139.6 & 221.0 \\
$I C 95$ & & 4.2 & 8.7 & 13.1 \\
\hline \hline
\end{tabular}

\section{NUMERICAL SIMULATIONS}

Apart from the experiments, we performed numerical simulations of the discharge of inert grains from a silo by gravity, following the same procedure as in Refs. [23,24,31]. We use softparticle molecular dynamics simulations of monosized disks in two dimensions. The restoring force in the normal direction of collision depends linearly on the particles overlap $\xi=d-r_{i j}$, with stiffness $k_{n}=10^{5}(\mathrm{mg} / \mathrm{d})$, where $d=1 \mathrm{~mm}$ is the particle diameter, $m=1$ is the mass, and $r_{i j}$ is the distance between the centers of the particles. Additionally, there is a dissipative force proportional to the relative normal velocity of the colliding grains, with damping parameter $\gamma_{n}=300(m \sqrt{g / d})$. We implement static friction by placing a spring in the direction tangential to the normal joining the centers of the particles. The elongation of this spring is obtained by integrating the relative velocity of the surfaces in contact. The parameters are $k_{t}=(2 / 7) k_{n}$ and $\gamma_{t}=200(m \sqrt{g / d})$. The friction coefficient is set to $\mu=0.5$. The number of particles varies from around 800 for the narrowest silo to more than 8000 for the widest.

The simulation protocol is as follows: A flat-bottomed rectangular silo is filled with grains which fall freely from a height much larger than their size. The side walls of the container are smooth, while the base is formed by fixed grains at mutual distances of $0.5 \mathrm{~d}$. Once all grains in the silo came to a rest, a hole is opened at the center of the base and the particles start to flow. The fallen grains are reinserted at the top of the silo in order to keep a constant height of the granular layer. A clog is detected when the kinetic energy of the system falls to the value it had before opening the hole (the difference amounts to several orders of magnitude) and remains under this value for 20000 time steps. Then, the avalanche size (measured in number of particles) and duration are registered and the flow is resumed by removing three of the grains forming the blocking arch.

\section{RESULTS}

The first experimental results presented are about the statistics of the avalanche sizes (measured in number of grains). In all the cases, for a given experimental condition, the avalanche size distribution has an exponential tail (not shown). Then, the first moment of the distribution, $\langle s\rangle$, is represented vs the silo width [Fig. 2(a)]. Unexpectedly, the dependence upon these parameters is nonmonotonic, displaying a minimum around 15-18 particle diameters. In order to rule out any uncontrolled experimental artifact, we implemented numerical simulations that revealed the same trend [Figs. 2(b) and 2(c)]. Undoubtedly, the avalanche size is minimum (and the probability of clogging maximum) for a silo width of 15-20 particle diameters. In addition, quantitative comparison of results displayed in Fig. 2 evidences the well-known strong effect that the outlet size has on the mean avalanche size [7-11], which is much stronger than that of the silo width. This issue impedes us from finding a dimensionless parameter that includes both $W$ and $D$ and collapses all the curves. To check if the nonmonotonic behavior is also present in three-dimensional silos, we have performed experiments with four different silo diameters $\left(W_{3 D}\right)$. The outcomes are summarized in Table I. Clearly, for values of $W_{3 D} / d_{3 D}$ above 5.75 the avalanche size grows with the silo width. Also, when the silo becomes very narrow (i.e., $W_{3 D} / d_{3 D}=4.5$ ), the avalanche size grows abruptly, up to the point that no clogging is observed after the discharge of more than $10^{4}$ grains. 

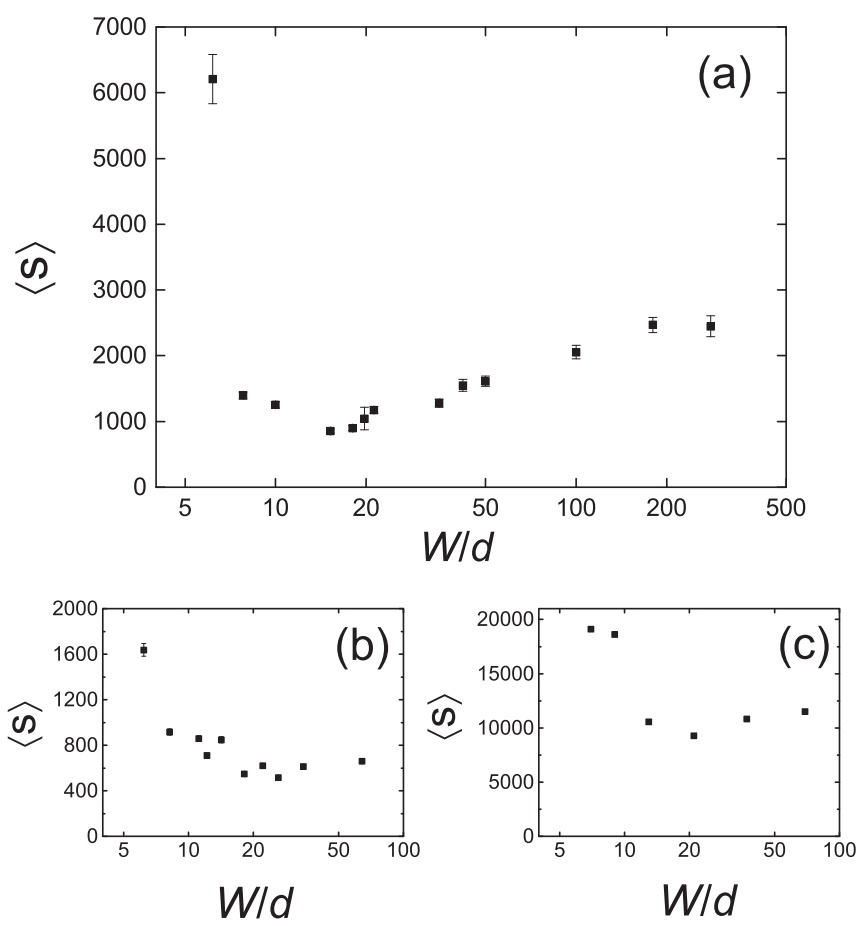

FIG. 2. Average avalanche size $\langle s\rangle$ in number of beads (\#) versus silo width $W$ rescaled by the particle size $d$. Results are presented for (a) experiments for an outlet size of $D / d=4.32$, (b) numerical simulations for $D / d=4.2$, and (c) numerical simulations for $D / d=5.0$. In all cases, the particle diameter is $d=1.00 \mathrm{~mm}$. Error bars indicate $95 \%$ confidence intervals. Note the lin-log scale.

Once we have shown that the nonmonotonicity of the avalanche size with the silo diameter is something generic, occurring for different outlet sizes and in two and three dimensions, we will focus on the $2 \mathrm{D}$ experiments, where we analyze several properties of the flow of grains

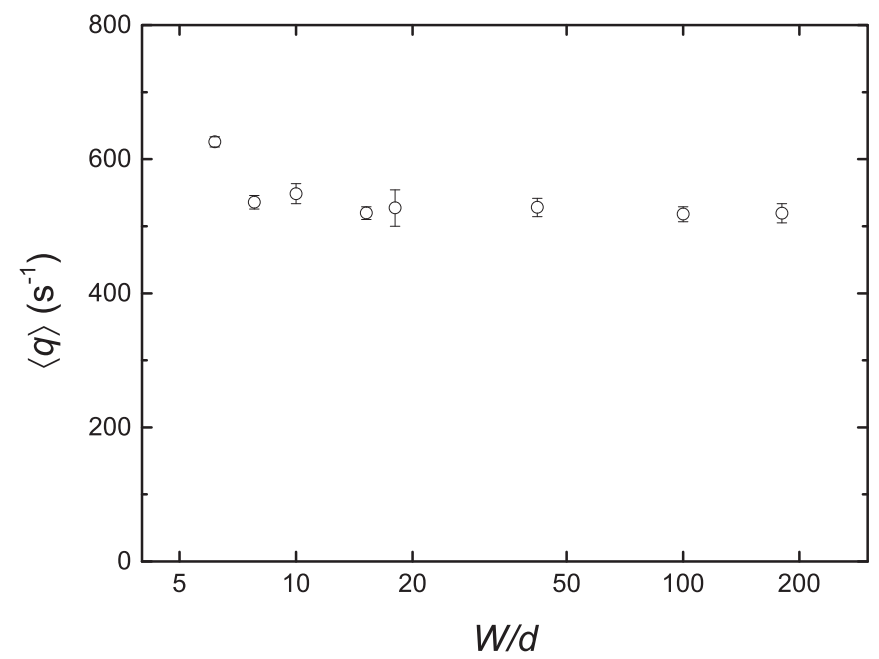

FIG. 3. Dependence of the flow rate $\langle q\rangle$ (measured in grains per second) on the rescaled silo width $\mathrm{W} / \mathrm{d}$ (note the lin-log scale). 


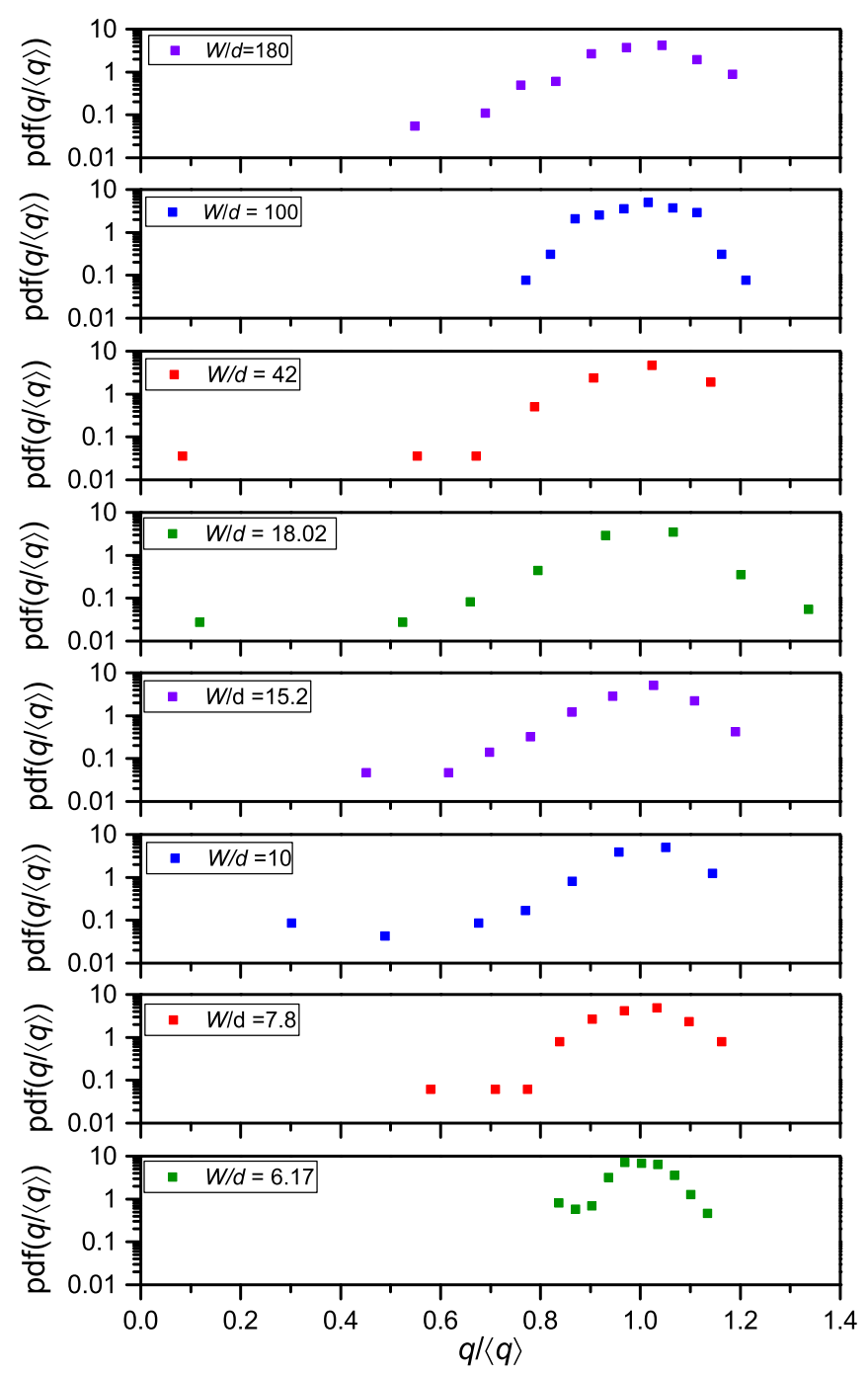

FIG. 4. Probability distribution function (pdf) of the instantaneous flow rates $q$ normalized by $\langle q\rangle$ for different rescaled silo widths $W / d$ as indicated in the legend. The values of $q$ are obtained by counting the number of particles passing through the outlet in temporal windows of $200 \mathrm{~ms}$.

through the bottleneck. The first one is the flow rate (Fig. 3), which seems to behave monotonically, increasing around $20 \%$ as the silo becomes narrower. By looking at the flow rate in small temporal windows (which we name instantaneous flow rate $q$ ) we can obtain the fluctuations of this magnitude (Fig. 4). Interestingly, for silo widths going from $W=10 \mathrm{~mm}$ to $W=42 \mathrm{~mm}$ (around the value corresponding to the minimum avalanche size), we observe the appearance of long tails toward small values of $q$, indicating that the intermittencies become more important. These long tails are not present for very narrow and wide silos, hence corroborating the existence of a relation between clogging and flow rate intermittencies [32,33].

The next step was to experimentally characterize, in the exit proximities, the solid fraction, velocity, and $\sigma^{k}$. The fields for six different silo widths are shown in Fig. 5. Reducing $W$ leads to a decrease of packing fraction in the lateral sides of the outlet, which seems to be related with a destabilization of the natural hopper that forms when the silo is wide enough. Also, as the silo 


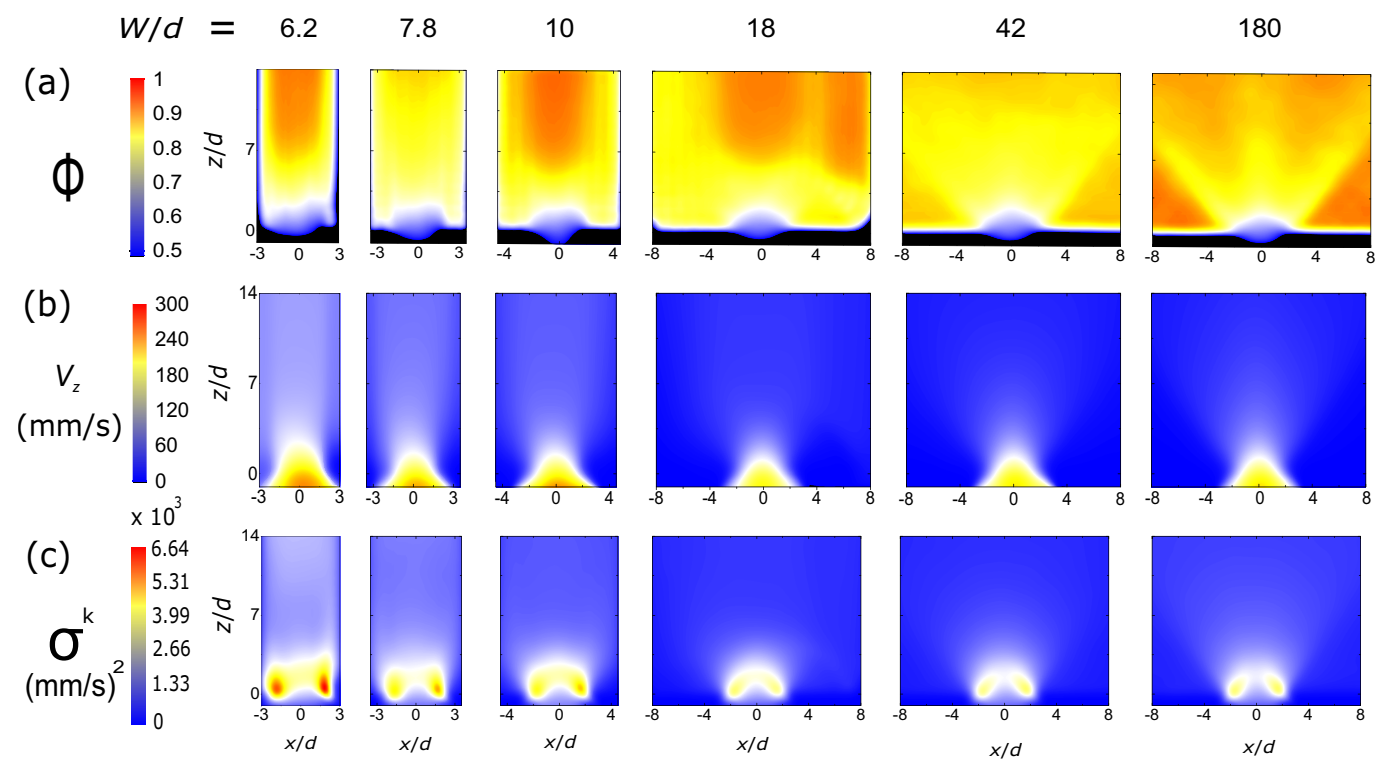

FIG. 5. Experimental fields of (a) two-dimensional packing fraction, (b) velocity, and (c) $\sigma^{k}$ in the outlet proximities for six silo widths as indicated at the top. The maps have been obtained from the particle positions and velocities after applying an averaging coarse-graining technique. For comparison, the same color-bar scales have been used in all cases.

narrows, the presence of the lateral wall near the orifice induces an increase of the vertical velocity at the center of the silo. Finally, the fields of $\sigma^{k}$ corroborate that the maximum values take place at an archlike region [29]. Noteworthy, as the silo width decreases, $\sigma^{k}$ increases, but also the arch shape becomes distorted.

Aiming to quantify these variables with more depth, we represent the solid fraction and vertical velocity versus the height within the silo, $z$, for a thin slice centered with the orifice, $x=0$ (Fig. 6).

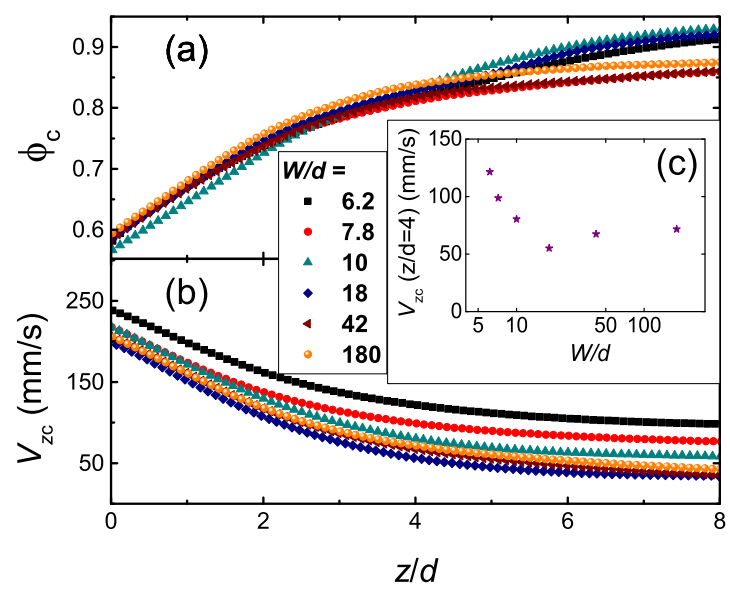

FIG. 6. (a) Packing fraction, $\phi_{c}$, and (b) vertical velocity, $v_{z c}$, at the center of the silo $(x=0)$ versus the height $z$ from the silo bottom. Experimental results are shown for six different silo widths as indicated in the legend. In panel (c), the value of $v_{z c}$ for $z=4 \mathrm{~mm}$ is plotted vs the silo width. In panel (a) some values marginally above the densest possible $2 \mathrm{D}$ packing are obtained due to slightly $3 \mathrm{D}$ arrangements. 

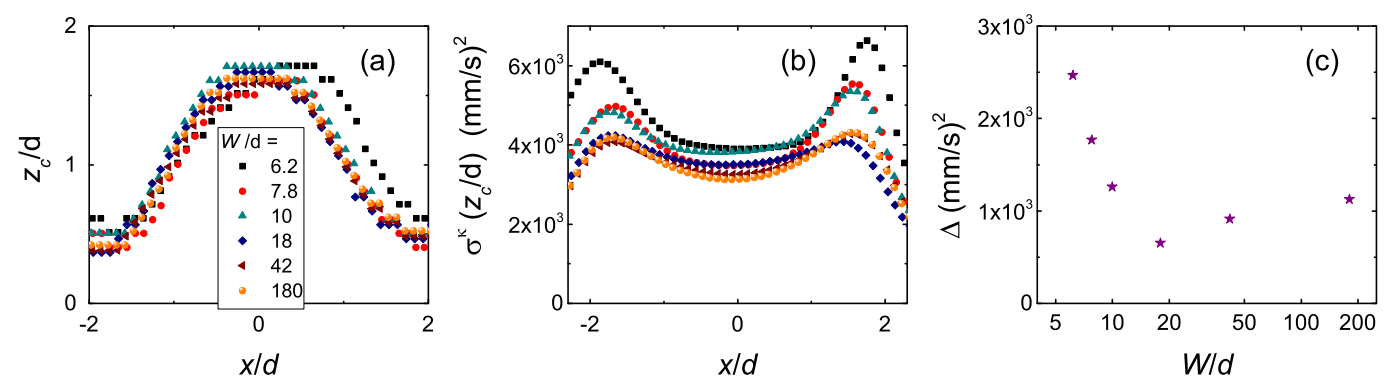

FIG. 7. (a) Position of $z_{c}$, the local maximum of $\sigma^{k}$ as a function of the distance to the center of the outlet $x$ for different silo widths as indicated in the legend. (b) Experimental values of $\sigma^{k}$ in the positions $z_{c}$ shown in panel (a). (c) Difference $\Delta$ between the maximum of $\sigma^{k}$ (for $x \approx 2 \mathrm{~mm}$ ) and the local minimum (at $x \approx 0 \mathrm{~mm}$ ) versus silo width. Note the lin-log scale.

Interestingly, $\phi$ does not show any clear trend that can be related with clogging. On the contrary, the velocity of the grains when $z>3 \mathrm{~mm}$ displays a nonmonotonic behavior that coincides with that of Fig. 2, being $v_{z}$ minimum for $W=18 \mathrm{~mm}$ [Fig. 6(c)]. This correlation might be related to the fact that as the kinetic energy of the particles when they form an arch increases, the more difficult it is for this structure to become stabilized, as suggested in Ref. [34] and numerically observed in Refs. [23,24].

Having discovered this possible relationship between clogging and particles velocity, it seems pertinent to further investigate other kinetic variables such as $\sigma^{k}$. In Fig. 7(a), we report the height $z_{c}$ at which the maximum value of $\sigma^{k}$ develops for each horizontal position, $x$. Clearly, this maximum has a characteristic arch shape [29], which seems to be independent on the silo width. In Fig. 7(b), the magnitude of $\sigma^{k}$ in the arch region is plotted as a function of $x$. The first noticeable result is that the maximum $\sigma^{k}$ always develops at the arch base $(x= \pm 2 \mathrm{~mm})$, whereas there is a local minimum at the center of the arch. Both magnitudes (the maximum and the minimum) monotonically reduce with the silo width. Therefore, this behavior can be connected with the strong reduction of avalanche size and flow rate observed with $W$ for narrow silos but not with the further increase of avalanche size observed for wider ones.

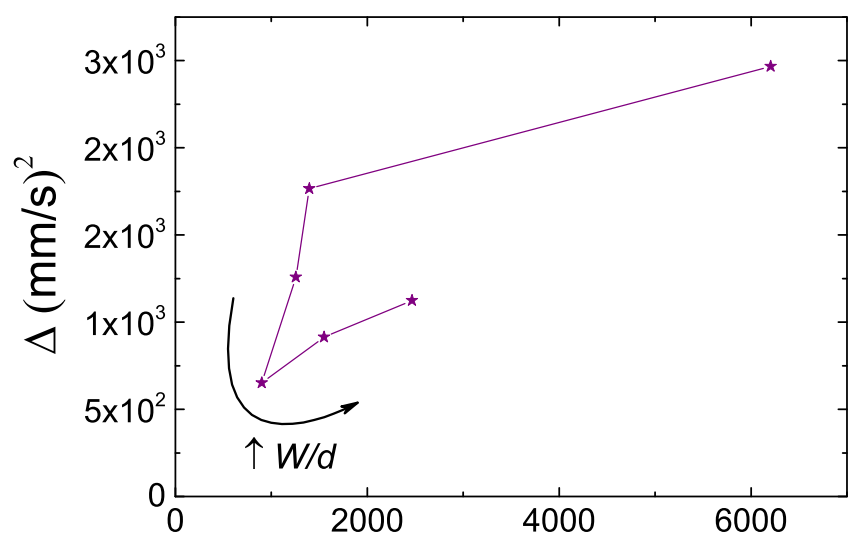

$\langle s\rangle$

FIG. 8. Difference $\Delta$ between the maximum of $\sigma^{k}$ (for $x \approx 2 \mathrm{~mm}$ ) and the local minimum (at $x \approx 0 \mathrm{~mm}$ ) vs the average avalanche size $\langle s\rangle$ (in number of beads). The arrow indicates the sense in which the silo width $W$ increases. 
Nevertheless, looking with more detail to the plots of Fig. 7(b), we observe that the 18-mm-wide silo (the one with the maximum probability of clogging) is the one in which $\sigma^{k}$ is more homogeneous along the arch. In order to obtain a simple measure of the $\sigma^{k}$ inhomogeneity along the arch, we have calculated $\Delta$, the difference in $\sigma^{k}$ between $x=0$ and $x= \pm 2 \mathrm{~mm}$. This magnitude is represented versus the silo width in Fig. 7(c), evidencing a strong resemblance with Fig. 2 and suggesting that the homogeneity of $\sigma^{k}$ in the arch region is important to clogging development. This is confirmed by plotting $\Delta$ vs $\langle s\rangle$ as in Fig. 8. Effectively, an increase of the mean avalanche (reduction of clogging probability) is associated with an enhancement of $\sigma^{k}$ inhomogeneity. Nonetheless, it is clear that there is not a one-to-one $\Delta-\langle s\rangle$ correspondence; indeed, at both sides of the minimum the relationship is different as, for the same increase of $\langle s\rangle$, the growth of $\Delta$ is more important for small values of $W$.

\section{CONCLUSIONS}

In summary, we have shown a previously unnoticed dependence of bottleneck clogging on silo width. The effect of this parameter is rather strong for narrow silos (where clogging increases with width) becoming weaker as the silo gets wider. In this region, clogging reduces with width, giving rise to a nonmonotonic behavior. This is associated with the effect that the silo width has on the velocity of grains in the region of arch formation and also with some features of the variance of the instantaneous velocity of the particles (which is proportional to the kinetic stress): Clogging is more likely when its magnitude is smaller and more homogeneous along the arch region.

Although more work is necessary to confirm this result for other silo widths and to unveil the origin of the changes in the kinematic variables, the importance of our findings is twofold. On the one hand, we connect clogging probability with a kinematic property of the flow of grains through the bottleneck; this suggests that analyzing the flowing regime can be used to predict whether or not an eventual arrest of the flow will develop. On the other hand, we prove the importance of silo width on clogging. It seems reasonable to think that the applied relevance of this finding is marginal, as most of real silos are wide enough to be in the asymptotic limit. Nevertheless, there are many other systems - such as particle rafts [35], pedestrian flows [36], and colloids [37]-where, commonly, the distance to the walls in the enclosure previous to the bottleneck is small and is therefore expected to affect clogging. Moreover, for the case of very narrow silos, this work raises new questions, such as if a small asymmetry of the outlet position is able to further prevent clogging or whether there is a smooth transition to a situation where the silo width equals the outlet size, i.e., the case of clogging in pipe flow $[38,39]$.

\section{ACKNOWLEDGMENTS}

We thank Raúl Cruz Hidalgo and Angel Garcimartín for useful comments. This work was funded by Ministerio de Economía y Competitividad (Spanish government) through Project No. FIS2014-57325.

[1] M. Delarue, J. Hartung, C. Schreck, P. Gniewek, L. Hu, S. Herminghaus, and O. Hallatschek, Self-driven jamming in growing microbial populations, Nat. Phys. 12, 762 (2016).

[2] M. D. Haw, Jamming, Two-Fluid Behavior, and Self-Filtration in Concentrated Particulate Suspensions, Phys. Rev. Lett. 92, 185506 (2004).

[3] D. Genovese and J. Sprakel, Crystallization and intermittent dynamics in constricted microfluidic flows of dense suspensions, Soft Matter 7, 3889 (2011).

[4] E. Dressaire and A. Sauret, Clogging of microfluidic systems, Soft Matter 13, 37 (2017). 


\section{GELLA et al.}

[5] D. Helbing, I. Farkas, and T. Vicsek, Simulating dynamical features of escape panic, Nature (London) 407, 487 (2000).

[6] I. Zuriguel, D. R. Parisi, R. C. Hidalgo, C. Lozano, A. Janda, P. A. Gago, J. P. Peralta, L. M. Ferrer, L. A. Pugnaloni, E. Clément, D. Maza, I. Pagonabarraga, and A. Garcimartín, Clogging transition of many-particle systems flowing through bottlenecks, Sci. Rep. 4, 7324 (2014).

[7] K. To, P. Y. Lai, and H. K. Pak, Jamming of Granular Flow in a Two-Dimensional Hopper, Phys. Rev. Lett. 86, 71 (2001).

[8] I. Zuriguel, A. Garcimartín, D. Maza, L. A. Pugnaloni, and J. M. Pastor, Jamming during the discharge of granular matter from a silo, Phys. Rev. E 71, 051303 (2005).

[9] K. To, Jamming transition in two-dimensional hoppers and silos, Phys. Rev. E 71, 060301(R) (2005).

[10] A. Janda, I. Zuriguel, A. Garcimartín, L. A. Pugnaloni, and D. Maza, Jamming and critical outlet size in the discharge of a two-dimensional silo, Europhys. Lett. 84, 44002 (2008).

[11] C. C. Thomas and D. J. Durian, Fraction of Clogging Configurations Sampled by Granular Hopper Flow, Phys. Rev. Lett. 114, 178001 (2015).

[12] I. Zuriguel, L. A. Pugnaloni, A. Garcimartín, and D. Maza, Jamming during the discharge of grains from a silo described as a percolating transition, Phys. Rev. E 68, 030301(R) (2003).

[13] L. Kondic, Simulations of two dimensional hopper flow, Gran. Matter 16, 235 (2014).

[14] T. Masuda, K. Nishinari, and A. Schadschneider, Critical Bottleneck Size for Jamless Particle Flows in Two Dimensions, Phys. Rev. Lett. 112, 138701 (2014).

[15] N. Roussel, T. L. H. Nguyen, and P. Coussot, General Probabilistic Approach to the Filtration Process, Phys. Rev. Lett. 98, 114502 (2007).

[16] C. C. Thomas and D. J. Durian, Geometry dependence of the clogging transition in tilted hoppers, Phys. Rev. E 87, 052201 (2013).

[17] S. Saraf and S. V. Franklin, Power-law flow statistics in anisometric (wedge) hoppers, Phys. Rev. E 83, 030301(R) (2011).

[18] J. Tang and R. P. Behringer, Orientation, flow, and clogging in a two-dimensional hopper: Ellipses vs. disks, Europhys. Lett. 114, 34002 (2016).

[19] T. Börzsönyi, E. Somfai, B. Szabó, S. Wegner, P. Mier, G. Rose, and R, Stannarius, Packing, alignment, and flow of shape-anisotropic grains in a 3D silo experiment, New J. Phys. 18, 093017 (2016).

[20] A. Ashour, S. Wegner, T. Trittel, T. Börzsönyi, and R. Stannarius, Outflow and clogging of shapeanisotropic grains in hoppers with small apertures, Soft Matter 13, 402 (2017).

[21] L. Pournin, M. Ramaioli, P. Folly, and Th. M. Liebling, About the influence of friction and polydispersity on the jamming behavior of bead assemblies, Eur. Phys. J. E 23, 229 (2007).

[22] S. Dorbolo, L. Maquet, M. Brandenbourger, F. Ludewig, G. Lumay, H. Caps, N. Vandewalle, S. Rondia, M. Mélard, J. van Loon, A. Dowson, and S. Vincent-Bonnieu, Influence of the gravity on the discharge of a silo, Gran. Matter 15, 263 (2013).

[23] R. Arévalo, I. Zuriguel, D. Maza, and A. Garcimartín, Role of driving force on the clogging of inert particles in a bottleneck, Phys. Rev. E 89, 042205 (2014).

[24] R. Arévalo and I. Zuriguel, Clogging of granular materials in silos: Effect of gravity and outlet size, Soft Matter 12, 123 (2016).

[25] S. Tewari, M. Dichter, and B. Chakraborty, Signatures of incipient jamming in collisional hopper flows, Soft Matter 9, 5016 (2013).

[26] M. Babic, Average balance equations for granular materials, Int. J. Eng. Sci. 35, 523 (1997).

[27] I. Goldhirsch, Stress, stress asymmetry, and couple stress: From discrete particles to continuous fields, Gran. Matt. 12, 239 (2010).

[28] T. Weinhart, C. Labra, S. Luding, and J. Y. Ooi, Influence of coarse-graining parameters on the analysis of DEM simulations of silo flow, Powder Tech. 293, 138 (2016).

[29] S. M. Rubio-Largo, A. Janda, D. Maza, I. Zuriguel, and R. C. Hidalgo, Disentangling the Free-Fall Arch Paradox in Silo Discharge, Phys. Rev. Lett. 114, 238002 (2015).

[30] R. Artoni and P. Richard, Average balance equations, scale dependence, and energy cascade for granular materials, Phys. Rev. E 91, 032202 (2015). 
[31] I. Zuriguel, A. Janda, A. Garcimartín, C. Lozano, R. Arévalo, and D. Maza, Silo Clogging Reduction By the Presence of an Obstacle, Phys. Rev. Lett. 107, 278001 (2011).

[32] A. Janda, R. Harich, I. Zuriguel, D. Maza, P. Cixous, and A. Garcimartín, Flow-rate fluctuations in the outpouring of grains from a two-dimensional silo, Phys. Rev. E 79, 031302 (2009).

[33] C. C. Thomas and D. J. Durian, Intermittency and velocity fluctuations in hopper flows prone to clogging, Phys. Rev. E 94, 022901 (2016).

[34] J. Koivisto and D. J. Durian, Friction controls submerged granular flows, Phys. Rev. E 95, 032904 (2017).

[35] A. Guariguata, M. A. Pascall, M. W. Gilmer, A. K. Sum, E. D. Sloan, C. A. Koh, and D. T. Wu, Jamming of particles in a two-dimensional fluid-driven flow, Phys. Rev. E 86, 061311 (2012).

[36] W. Liao, A. Tordeux, A. Seyfried, M. Chraibi, K. Drzycimski, X. Zheng, and Y. Zhao, Measuring the steady state of pedestrian flow in bottleneck experiments, Phys. A (AMsterdam, Neth.) 461, 248 (2016).

[37] U. Zimmermann, F. Smallenburg, and H. Löwen, Flow of colloidal solids and fluids through constrictions: Dynamical density functional theory versus simulation, J. Phys.: Condens. Matter 28, 244019 (2016).

[38] A. Janda, I. Zuriguel, A. Garcimartín, and D. Maza, Clogging of granular materials in narrow vertical pipes discharged at constant velocity, Gran. Matter 17, 545 (2015).

[39] F. Verbucheln, E. J. R. Parteli, and T. Pöschel, Helical inner-wall texture prevents jamming in granular pipe flows, Soft Matter 11, 4295 (2015). 